\title{
BRIEF ARTICLE
}

\section{Coexisting Pediatric Acute Generalized Exanthematous Pustulosis and Staphylococcal Scalded Skin Syndrome}

\author{
Cassandra Beard, DO, MPH${ }^{1}$, Rafael Mojica, BS², Sarah Ferrer-Bruker, DO1, Karthik \\ Krishnamurthy, DO 1 \\ ${ }^{1}$ Division of Dermatology, HCA Healthcare/Mercer University School of Medicine/Orange Park Medical Center \\ Program, Orange Park, FL \\ ${ }^{2}$ Edward Via College of Osteopathic Medicine, Spartanburg, SC
}

\section{ABSTRACT}

Acute Generalized Exanthematous Pustulosis is a rare drug-induced skin disorder that can present at any age. It is typically noted by swelling and erythema, with numerous facial and/or anogenital nonfollicular pustules that quickly disseminate. Staphylococcal scalded skin syndrome presents with erythema and swelling that similarly favor the head and intertriginous sites with subsequent bullae formation. We present a case of a four-year-old female who presented with SSSS complicated by the development of AGEP and discuss the course of her condition and treatment.

\section{INTRODUCTION}

Acute generalized exanthematous pustulosis (AGEP) is a disease of acute onset, typically following drug intake. Patients with AGEP develop an abrupt febrile eruption of pinhead-sized nonfollicular pustules on an erythematous base favoring the face or intertriginous areas. The lesions quickly spread within hours to involve the trunk and limbs. ${ }^{1}$ Staphylococcal scalded skin syndrome (SSSS) presents in a similar distribution with swelling and erythema with subsequent bulla formation and exfoliation. ${ }^{2}$ Although rare, SSSS most commonly presents in the pediatric population with an annual incidence of 7.67 per million U.S. children. ${ }^{3}$ There are far fewer reported cases of AGEP in the pediatric population, which is highlighted by the paucity of such incidence reports. ${ }^{4}$ We present a case of a four-yearold female whose diagnosis was complicated by overlapping features of both SSSS and AGEP.

\section{CASE REPORT}

A previously healthy four-year-old female presented to her pediatrician with a fever of $101^{\circ} \mathrm{F} / 38^{\circ} \mathrm{C}$, facial edema, and a shiny erythematous eruption of her face, neck, and shoulders that spread to the neck and axilla overnight. She then developed rhinorrhea and shallow perinasal erosions with yellow crusting, leading to a diagnosis of bullous impetigo. She was given topical mupirocin ointment and oral amoxicillin. The erythema continued to spread across the chest, down the back, and across the face with areas of vesiculation that ruptured, leading to crusts and scaling. She also developed radial fissuring around the mouth and eyes, clinically consistent with staphylococcal scalded skin. The following day, the exanthem evolved into a different

March 2021 Volume 5 Issue 2 


\section{SKIN}

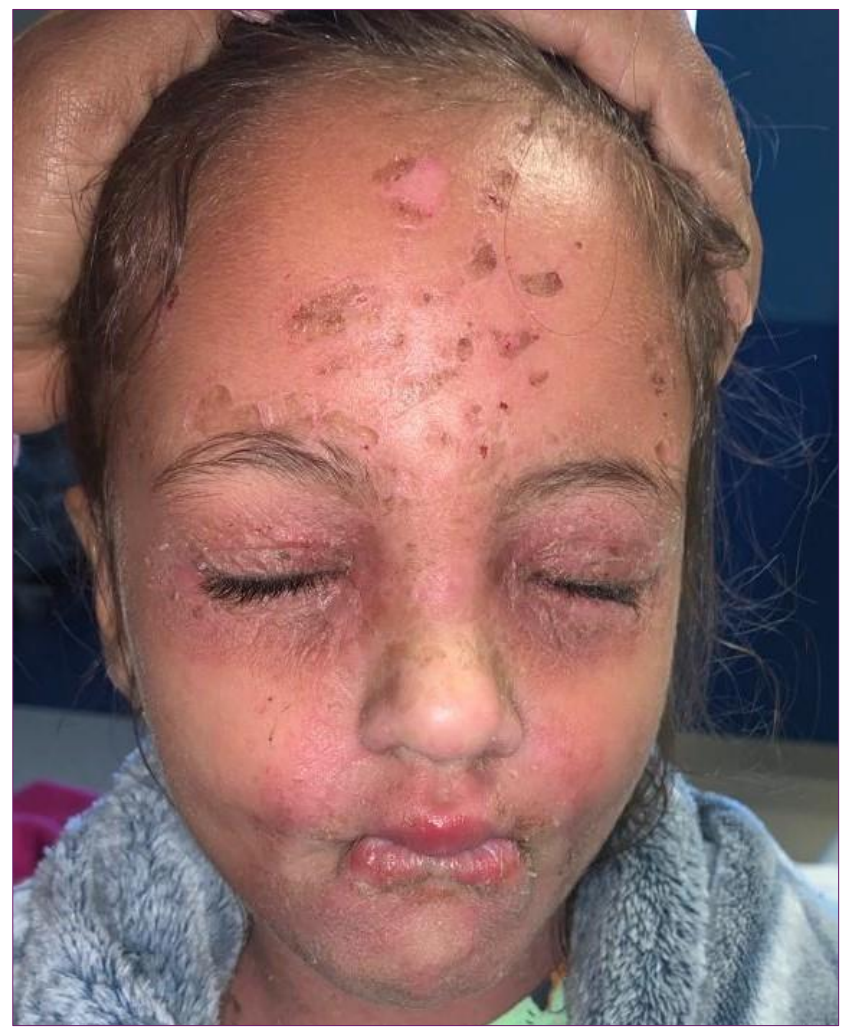

Figure 1. Paranasal, periorbital, and frontal erosions with yellow crusting and desquamation.

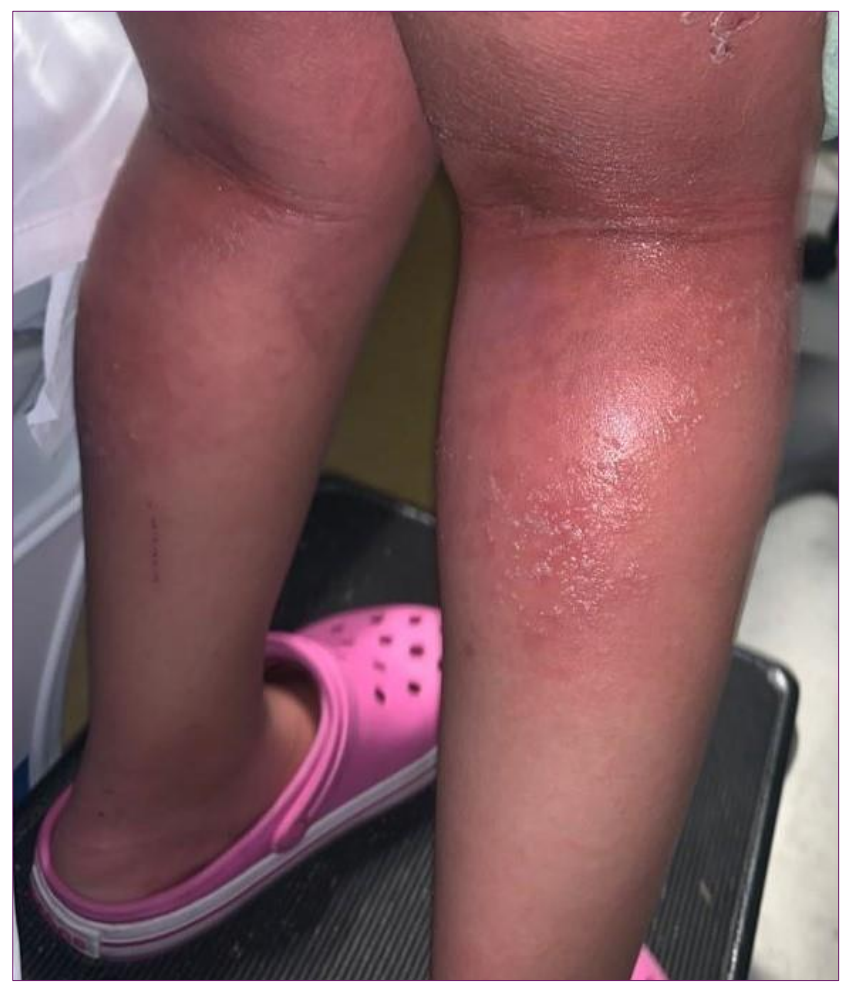

Figure 2. Popliteal advancing erythema featuring nonfollicular pustules at the edge. morphology. Nonfollicular pustules developed at the advancing edge of the erythema. A wound culture of the pustules was negative for bacterial growth. A shave biopsy of the right thigh demonstrated subcorneal pustules with eosinophils, consistent with AGEP. ${ }^{5}$ Amoxicillin was discontinued and treatment with vancomycin initiated.

Upon discharge two days later, the patient completed a short course of oral cephalexin and TID application of triamcinolone cream $0.1 \%$ with complete resolution of skin lesions.

\section{DISCUSSION}

The subcorneal pustules are a common histological feature of both SSSS and AGEP [6]. In our case, the presence of an inflammatory infiltrate favors AGEP, as SSSS typically lacks inflammatory cells both in the bullae and in the underlying dermis. ${ }^{7}$ In SSSS, the exfoliative toxins A and/or B released from Staphylococcus aureus attack desmoglein 1, resulting in loss of adhesion of keratinocytes in the granular layer of the epidermis without apoptosis and does not elicit an inflammatory response. ${ }^{8,9}$ Therefore, in SSSS, early treatment with antibiotics is prudent. Additional supportive care with fluid and electrolytes might result in quick recovery. Patient might under short hospitalization, if intravenous antibiotics are necessary, and is advised to continue treatment for up to 15 days.

In cases of AGEP, treatment with oral antibiotics do not provide significant improvement, and side effects from antibiotic treatment may worsen the patient's condition. ${ }^{1}$ If the source of treatment is a cutaneous infection, topical antibiotics may be an acceptable alternative in a non-toxic 
pediatric patient. However, early treatment with systemic antibiotics can be lifesaving for deteriorating patients who may have disseminated staphylococcus or streptococcus infection. While AGEP is not associated with high rates of mortality, it is important to control in young, elderly, immunocompromised, or pregnant patients. ${ }^{8}$ Alternatively, in most cases of AGEP, withdrawal of the offending drug and supportive care results in recovery. Moist dressings and antiseptics can be considered during the pustular phase. In rare but severe cases, infliximab and etanercept have been shown to rapidly stop the pustulation and hasten recovery. In case pruritus is a prominent symptom topical steroids and other anti-pruritic creams (e.g. camphor/menthol) may be helpful. Oral steroids have not been shown to shorten the overall duration of the condition or improve outcomes. If a drug reaction is suspected, patch testing can be considered. ${ }^{10}$

\section{CONCLUSION}

The presentation of SSSS and AGEP can be differentiated clinically at their initial presentation but may appear very similar in their later stages. Early on the disease process, SSSS can be distinguished from AGEP by the presence of a scarlatiniform rash and desquamation in areas of friction e.g. the flexural folds in the absence of an iatrogenic exposure. ${ }^{5}$ Although there have been rare reports of an infectious etiology in AGEP, most cases have been diagnosed by the onset of symptomatology within 24-48 hours following drug intake. ${ }^{6}$ The above case is interesting due to the primary diagnosis of SSSS but with the complicated development of nonfollicular pustules along the advancing edge of lesional erythema following amoxicillin exposure and subsequent histological confirmation of
AGEP. Histologic features of AGEP include the presence of a mixed interstitial and middermal perivascular infiltrate along with necrotic keratinocytes and the absence of tortuous or dilated blood vessels, whereas the subcorneal pustules of SSSS are not associated with an inflammatory infiltrate ${ }^{[1]}$. Once a diagnosis of SSSS or AGEP has been made, it is important to choose the appropriate antibiotic therapy and supportive treatment for each disease and patient population.

Conflict of Interest Disclosures: None

Funding: None

Corresponding Author:

Cassandra Beard, DO, MPH

Division of Dermatology

HCA Healthcare/Mercer University School of Medicine/Orange Park Medical Center Program 2001 Kingsley Ave

Orange Park, FI 32073

Phone: 573-301-9744

Email: cibeard@atsu.edu

\section{References:}

1. Sidoroff A, Dunant A, Viboud C, Halevy S, Bavinck JB, et al. (2007) Risk factors for acute generalized exanthematous pustulosis (AGEP) results of a multinational case-control study (EuroSCAR). Br J Dematol 157: 989-996.

2. Napoli B, D'Arpa N, D'Amelio L, et al. Staphylococcal Scalded Skin Syndrome: Criteria for Differential Diagnosis from Lyell's Syndrome. Two Cases in Adult Patients. Ann Burns Fire Disasters. 2006;19(4):188-191.

3. Staiman, A., Hsu, D. and Silverberg, J. (2018), Epidemiology of staphylococcal scalded skin syndrome in U.S. children. Br J Dermatol, 178: 704-708.

4. Davidovici B, Dodiuk-Gad R, Rozenman D. The Profile of Acute Generalized Exanthematous Pustulosis in Israel During the Period 2002-2005. IMAJ 2008: 10: June: 410-412

5. Henry SM, Stanfield MM, Dorey HF (2019) Pediatric Acute Generalized Exanthematous Pustulosis Involving Staphylococcal ScarletFever. Med Case Rep Vol. 5 No.1:88.

6. Szatkowski J, Schwartz RA. Acute generalized exanthematous pustulosis (AGEP): A review and 
update. Journal of the American Academy of Dermatology. Mosby Inc (2015) P $843-848$

7. De A, Das S, Sarda A, Pal D, Biswas P. Acute Generalised Exanthematous Pustulosis: An Update. Indian J Dermatol. 2018;63(1):22-29.

8. Mishra AK, Yadav P, Mishra A. A Systemic Review on Staphylococcal Scalded Skin Syndrome (SSSS): A Rare and Critical Disease of Neonates. Open Microbiol J. 2016;10:150-159 $P$.

9. Finlay, A. and Patel, G. (2019). Staphylococcal scalded skin syndrome: diagnosis and management. J Clin Dermatol (2003) 4: 165

10. 10. Pecina JL, Cappel MA. Acute generalized exanthematous pustulosis. Skinmed 8(4), 210$214 \mathrm{P}$ 